\title{
Choosing a Fluorescence Microscopy Imaging Modality for Live Quantitative Experiments
}

\author{
Talley J. Lambert ${ }^{1}$ and Jennifer C. Waters ${ }^{1}$ \\ ${ }^{1}$ Harvard Medical School/Department of Cell Biology, Boston, MA USA
}

There is no one fluorescence microscopy imaging modality that is "best" for quantitative imaging experiments. Instead, the modality that will yield the most accurate, precise and biologically relevant results depends heavily on the specimen and the experimental design. Common fluorescence microscopy imaging modalities (Fig. 1), including widefield epi-fluorescence, point scanning confocal, spinning disk confocal and total internal reflection fluorescence (TIRF), yield very different images of the same specimen with regards to signal-to-noise ratio and signal-to-background ratio (contrast), and therefore choice of imaging modality will affect quantitative measurements. Acquiring images for quantitative analysis of live specimens usually requires compromises in image quality to accommodate addition needs of the specimen and experiment, including minimizing photo-toxicity and photo-bleaching and achieving adequate temporal resolution. Guidelines for choosing and comparing modalities will be given, as well as suggestions for identifying and addressing the common problems associated with using each modality for quantifying fluorescence intensity.

Figure 1. Mammalian cells expressing mEmerald-Eb1, imaged with (A) widefield fluorescence, (B) spinning disk confocal, (C) point scanning confocal, and (D) TIRF microscopy. All images were collected with a Nikon Plan Apo 60x 1.4 lens and are displayed at the same magnification. Scalebar in (D) $=15 \mu \mathrm{m}$.
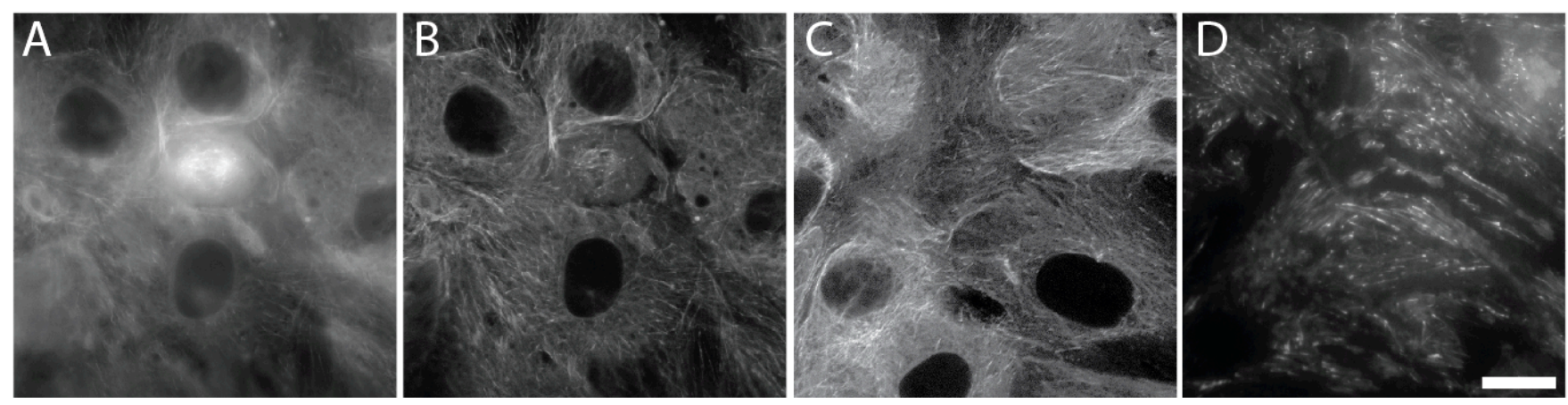